\title{
Factors that promote Bystander intervention in Cases of Sexual Harassment
}

\author{
Avigail Moor \\ Tel Hai College
}

\begin{abstract}
.
Sexual harassment of women occurs at alarmingly high rates, which have remained steady irrespective of the increased societal awareness of this problem. It appears that all the public discourse on this topic, including research, prevention programs, and public campaigns have not made a noticeable dent in this prevalence. This calls for new course of action. Raising awareness regarding the preventive role of bystanders might be it. To that end, the present study sought to establish what promotes bystander intervention and what hinders it. Three hundred and twenty-four men and women, ranging in age from 20-40, participated in this study, completing self-report questionnaires on the topics under investigation. Results indicated that the proclivity to intervene as a bystander is impacted by various factors. The most consequential among them is gender, with twice as many women as men, $70 \%$ vs $38 \%$ respectively, being positively inclined to take action in such cases. Other significant factors included belief in rape myths and having empathy towards perpetrators, which reduced the likelihood of bystander intervention. Holding the attitude that it is possible to freely consent to sex while intoxicated had a similar impact. The discussion addresses various preventive implications.
\end{abstract}

Keywords - Bystander intervention, Sexual harassment prevention, Sexual Harassment, Rape Myths 